\title{
GENERIC WELL POSEDNESS OF SUPINF PROBLEMS
}

\author{
P.S. KENDEROV AND R.E. LUCCHETTI
}

\begin{abstract}
We consider two notions of well posedness for problems of the type $\sup _{X} \inf _{Y} f(x, y)$ and give conditions under which the majority (in Baire category sense) of bounded functions $f$ defined in $X \times Y$ give rise to problems which are well posed. As a corollary we get that the problem $\sup _{x} f(x)$ is well posed for the majority of bounded lsc real valued functions $f$ if, and only if, $X$ contains a dense completely metrisable subset.
\end{abstract}

\section{INTRODUCTION AND PRELIMINARY REMARKS}

Let $X, Y$ be topological spaces and $K: X \rightarrow Y$ a set-valued mapping with $K x \neq \emptyset$ for all $x \in X$. Denote by $B(X \times Y)$ the space of all bounded real-valued functions $f(x, y)$ defined in $X \times Y$. Every $f \in B(X \times Y)$ generates the problem

$$
\sup _{x \in X} \inf _{y \in K x} f(x, y)
$$

This problem arises, for instance, in connection with the following two players game. The first player (sometimes called the "leading player") selects points $x \in X$ and wants to maximise the profit function $f(x, y)$. The latter function depends also on the choices $y$ of the second player. Once the leading player selects some $x^{\prime} \in X$ the "move" of the second player is to select some $y$ in the set of all admissible choices: $y \in K x^{\prime} \subset Y$. Then the value $v_{f}:=\sup _{X} \inf _{y \in K x} f(x, y)$ shows the maximal profit that can be guaranteed for the leading player even if the second player behaves in the "most unfriendly way" by always selecting $y^{\prime} \in K x^{\prime}$ so that $f\left(x^{\prime}, y^{\prime}\right)=\inf _{y \in K x^{\prime}} f\left(x^{\prime}, y\right)$.

In the case when, for every $x^{\prime}$, the set $K x^{\prime}$ is determined by some optimisation problem, for instance as $K x^{\prime}:=\left\{y^{*} \in Y: g\left(x^{\prime}, y^{*}\right)=\inf _{Y} g\left(x^{\prime}, y\right)\right\}$ where $g$ is a given

Received 23rd August, 1995.

Paper prepared during the visit of the first author to the Department of Mathematics of Milano and a visit of the second author to the Institute of Mathematics of the Bulgarian Academy of Sciences, in the setting of a scientific agreement between the Bulgarian Academy of Sciences and the Italian CNR. The first author was also supported by the Bulgarian National Foundation for Scientific Research under contract MM - 408/94.

Copyright Clearance Centre, Inc. Serial-fee code: 0004-9729/96 \$A2.00+0.00. 
function from $B(X \times Y)$, the problem $\left(P_{f}\right)$ is known as the "two level optimisation problem" or "Stackelberg problem" (for more information in this direction see [24, 1, $17,15])$.

In the partial case when $K x=Y$ for all $x \in X$ the problem $\left(P_{f}\right)$ is still interesting, at least in connection with the study of saddle points for the function $f(x, y)$.

The point $\left(x_{0}, y_{0}\right) \in X \times Y$ is called a solution to $\left(P_{f}\right)$ if $f\left(x_{0}, y_{0}\right)=\inf _{y \in K x_{0}} f\left(x_{0}, y\right)$ $=v_{f}$. The set of all such solutions will be denoted in this paper by $S(f)$. The set $S(f)$ may be empty for some $f \in B(X \times Y)$.

From the point of view of the first player in the above game a solution to $\left(P_{f}\right)$ is any point $x_{0} \in X$ for which $\inf _{y \in K x_{0}} f\left(x_{0}, y\right)=v_{f}$. To underline the difference between these two notions we say that the point $x_{0} \in X$ is a "sup-solution" of $\left(P_{f}\right)$ if $w_{f}\left(x_{0}\right)=$ $\sup _{X} w_{f}(x)$, where $w_{f}(x):=\inf _{y \in K x} f(x, y)$. The set of all such "sup-solutions" to $\left(P_{f}\right)$ will be denoted by $M(f)$. Clearly, if $\left(x_{0}, y_{0}\right) \in S(f)$, then $x_{0} \in M(f)$. It may however happen that $M(f) \neq \emptyset$ but $S(f)=\emptyset$.

The sequence $\left(x_{i}\right)_{i \geqslant 1} \subset X$ is called maximising for $\left(P_{f}\right)$ if $\lim _{i \rightarrow \infty} w_{f}\left(x_{i}\right)=v_{f}$.

The problem $\left(P_{f}\right)$ will be called sup-well-posed (sup-WP) if every maximising sequence converges to some sup-solution of $\left(P_{f}\right)$ (in this case $\left(P_{f}\right)$ has a unique supsolution). That is, $\left(P_{f}\right)$ is sup-WP if and only if the function $w_{f}(x)$ is Tykhonov well posed (T-WP) (see $[\mathbf{9}, \mathbf{2 5}])$.

The sequence $\left(x_{i}, y_{i}\right)_{i \geqslant 1} \subseteq X \times Y$ is called optimising for $\left(P_{f}\right)$ if

(i) $y_{i} \in K x_{i}$ for $\mathrm{i}=1,2,3, \ldots$;

(ii) $\lim _{i \rightarrow \infty} w_{f}\left(x_{i}\right)=v_{f}$ (that is, $\left(x_{i}\right)_{i \geqslant 1}$ is maximising for $\left(P_{f}\right)$ );

(iii) $\lim _{i \rightarrow \infty} f\left(x_{i}, y_{i}\right)=v_{f}$.

The problem $\left(P_{f}\right)$ is called well posed (WP) if every optimising sequence $\left(x_{i}, y_{i}\right)_{i \geqslant 1}$ converges to some solution $\left(x_{0}, y_{0}\right)$ of $\left(P_{f}\right)$. (In this case $\left(P_{f}\right)$ has a unique solution.)

If $\left(P_{f}\right)$ is WP, then it is also sup-WP. Simple examples show that $\left(P_{f}\right)$ may be sup-WP without being WP.

Let $E$ be a closed subset of $(B(X \times Y),\|\cdot\|)$, where $\|f\|=\sup _{X \times Y} \mid f(x, y) \|$. Then $E$ is a complete metric space and the following definition makes sense.

DEFINITION 1.1: We say that $E$ is generically sup-WP (generically WP) if there is a dense $G_{\delta}$ subset $G \subset E$ such that the problem $\left(P_{f}\right)$ is sup-WP (WP) for every $f \in G$.

In what follows we present a general scheme which allows us to show that many particular subsets $E$ of $B(X \times Y)$ are generically sup-WP or generically WP. We demonstrate this in the three (most important for us) cases when $E$ coincides with the 
space $C(X \times Y)$ of all bounded continuous functions in $X \times Y$ or the space $L(X \times Y)$ of all bounded lower semicontinuous functions in $X \times Y$ or the space $U(X \times Y)$ of all bounded upper semicontinuous functions in $X \times Y$.

In particular, we prove (see Theorem 3.7) that, if $K: X \rightarrow Y$ is upper semicontinuous (usc) with compact images then the following assertions are equivalent:

(a) $X$ contains a dense completely metrisable subset;

(b) $C(X \times Y)$ is generically sup-WP;

(c) $L(X \times Y)$ is generically sup-WP.

In the partial case when $Y$ is a singleton the space $C(X \times Y)$ (the space $L(X \times Y))$ can be identified with the space $C(X)(L(X))$ of all bounded continuous (lower semicontinuous) functions in $X$. Sup-well-posedness in this case is just the Tykhonov well posedness of the corresponding function. We get from Theorem 3.7 in this case that condition (a) is equivalent to each of the following two statements:

$\left(\mathrm{b}^{\prime}\right), C(X)$ is generically Tykhonov WP;

(c') $L(X)$ is generically Tykhonov WP.

Thus our result contains as a partial case Theorem 3.5 from [6] where the equivalence between (a) and ( $\left.b^{\prime}\right)$ was proved. (For compact spaces $X$ this equivalence was established in [4] and [5].)

For other classes of set-valued mappings $K$ (not necessarily upper semicontinuous) we show that, if $K$ is lower semicontinuous and $X$ is a complete metric space, then $C(X \times Y)$ is generically sup-WP. (See Theorem 3.10 for a more general statement.)

Concerning generic well posedness the scheme we use allows us to show that, if $X$ contains a dense completely metrisable subset, $Y$ is a complete metric space and $K: X \rightarrow Y$ is usc and compact-valued, then both $C(X \times Y)$ and $L(X \times Y)$ are generically WP (see Theorem 4.3).

The attempt to make this result symmetric with respect to the requirements imposed on $X$ and $Y$ fails. Simple examples (in which $X$ is a singleton) show that the result is not true for spaces $Y$ which contain a dense completely metrisable subset. This is actually not strange because the space $Y$ enters into our problem only via the mapping $K$ and the latter may send the points of $X$ into the "nonmetrisable part" of $Y$. This suggests that the generic well posedness of $\left(P_{f}\right)$ depends primarily on the mapping $K$ (on the topological structure of its graph). In this direction we have the following statement (see Theorem 4.4): Let $K: X \rightarrow Y$ be lsc, usc and compact-valued. Then the following are equivalent

(d) $C(X \times Y)$ is generically WP;

(e) $\operatorname{Gr} K:=\{(x, y) \in X \times Y: y \in K x\}$ contains a dense completely metrisable subset. 
Note that condition (e) is satisfied if both $X$ and $Y$ contain dense completely metrisable subsets and $K x=Y$ for every $x \in X$. (In this case $g r K=X \times Y$.) In particular, one derives that for the majority of functions $f \in C(X \times Y)$ the problem

$$
\sup _{X} \inf _{Y} f(x, y)
$$

is well posed, provided $Y$ is compact and both $X$ and $Y$ contain dense completely metrisable subsets. This improves significantly the main result from [14].

The scheme we use to prove all these statements has its roots in the general approach developed in $[4,5,6,13]$ for the study of generic well-posedness of simple (one level) optimisation problems generated by continuous functions. Following this approach we present in the next section some general results concerning generic singlevaluedness and generic continuity of set-valued mappings. These results are then applied in Sections 3 and 4 to the mappings $M$ and $S$ in order to establish that, under some conditions, these mappings are generically single-valued and usc. It then remains to observe that at the points where $M$ and $S$ are single-valued and usc we have supwell-posedness and well posedness respectively.

An equivalent form of the well posedness of $\left(P_{f}\right)$ was used in [17]. Close in spirit to this definition are also the notions studied in $[19, \mathbf{2 6}, \mathbf{2 7}]$. There are many other results concerning genericity of well posed (one-level) problems. Among them we recall $[2,3,7,8,10,12,18,21,22]$.

\section{Generic Properties of SET-VAlued Mappings}

In this section we present some properties of the set-valued mappings which are at the base of our results concerning generic sup-well posedness (generic well posedness).

DEFINITION 2.1: [11] The topological space $Z$ is said to be fragmented by the metric $d\left(z_{1}, z_{2}\right)$ (which is defined on $Z$ ) if for every $\varepsilon>0$ and every nonempty set $A \subset Z$ there exists a nonempty set $A^{\prime} \subset A$ such that $d-\operatorname{diam} A^{\prime} \leqslant \varepsilon$ and $A^{\prime}$ is relatively open in $A$ (that is, there is some open set $V \subset Z$ such that $A^{\prime}=V \cap A$ ).

Of special interest for us will be spaces $Z$ which are fragmented by a complete metric $d(\cdot, \cdot)$ which generates a metric topology stronger or equal to the topology of $Z$.

Important examples of fragmentable space are the spaces homeomorphic to a weakly compact subset of a Banach space or to a weak* compact subset of a dual Banach space with the Radon-Nikodym Property. In both cases the fragmenting metric is generated by the corresponding norm (for more information see $[\mathbf{2 0}, \mathbf{2 3}]$ ).

Let $E$ and $Z$ be topological spaces and $F: E \rightarrow Z$ be a set-valued map. By Dom $F$ we denote the set $\{x \in E: F x \neq \emptyset\}$. 
Definition 2.2: [13] The mapping $F$ is called minimal at some $x_{0} \in E$ if for every open $V \subset Z$ with $F\left(x_{0}\right) \cap V \neq \emptyset$ there exists an open set $U \subset E$ which contains $x_{0}$ in its closure $\bar{U}$ and is such that $F(U):=\bigcup\{F(x): x \in U\} \subset V . F$ is called minimal if it is minimal at each point of $E$.

The map $F: E \rightarrow Z$ is minimal if and only if for every pair of open sets $U_{1} \subset E$, $V \subset Z$ with $F\left(U_{1}\right) \cap V \neq \varnothing$ there is some open set $U_{2} \neq \emptyset, U_{2} \subset U_{1}$, such that $F\left(U_{2}\right) \subset V$.

Definition 2.3: $F: E \rightarrow Z$ is called upper semicontinuous (usc) at $x_{0} \in E$ if for every open $V \subset Z$ with $F x_{0} \subset V$ there exists some open $U \ni x_{0}$ such that $F(U) \subset V$. If $F$ is usc at each point of $E$, we shall simply say that $F$ is usc.

Theorem 2.4. Let $E$ be a complete metric space and let $Z$ be fragmented by some metric $d(\cdot, \cdot)$. Let $F: E \rightarrow Z$ be a minimal mapping with $\operatorname{Dom} F$ dense in $E$. Then there exists a dense $G_{\delta}$ set $G \subset E$ such that for every $x_{0} \in G$ a sequence $\left(U_{n}\right)_{n \geqslant 1}$ of open sets $U_{n} \ni x_{0}$ exists with

$$
\lim _{i \rightarrow \infty}\left(\operatorname{diam} U_{i}\right)=0 \text { and } \lim _{i \rightarrow \infty}\left(d-\operatorname{diam} F\left(U_{i}\right)\right)=0
$$

ProOF: For every positive integer $n$ consider the open set $H_{n}=\bigcup\{U: U$ open in $E, \operatorname{diam} U \leqslant 1 / n$ and $\operatorname{diam} F(U) \leqslant 1 / n\}$. We show that $H_{n}$ is dense in $E$. Take some open $U \subset E, U \neq \emptyset$. Since $\operatorname{Dom} F$ is dense in $E$, the set $A:=F(U) \neq \emptyset$. Fragmentability of $Z$ implies that there exists some open $V \subset Z$ such that $A^{\prime}:=$ $V \cap A \neq \emptyset$ and $\operatorname{diam} A^{\prime}<1 / n$. By minimality of $F$ there exists some open $U^{\prime} \neq \emptyset$, $U^{\prime} \subset U$, such that $F\left(U^{\prime}\right) \subset V$. Then $F\left(U^{\prime}\right) \subset V \cap F(U)=A^{\prime}$. Hence $\operatorname{diam} F\left(U^{\prime}\right)<$ $1 / n$. Without loss of generality we can suppose that $\operatorname{diam} U^{\prime}<1 / n$. Therefore $\emptyset \neq$ $U^{\prime} \subset H_{n} \cap U$.

The set $G=\bigcap_{n \geqslant 1} H_{n}$ is dense $G_{\delta}$ in $E$. For every $x_{0} \in G$ there is a sequence of open sets $U_{n} \ni x_{0}$ such that $\operatorname{diam} U_{n} \leqslant 1 / n$ and $\operatorname{diam} F\left(U_{n}\right) \leqslant 1 / n$.

CoRollary 2.5. Under the hypotheses of Theorem 2.4 the map $F$ is singlevalued and usc at any point $x_{0} \in \operatorname{Dom} F \cap G$ with respect to the topology generated in $Z$ by the metric $d$.

Definition 2.6: The map $F: E \rightarrow Z$ is called open if $F(U)$ is an open subset of $Z$ whenever $U$ is an open subset of $E$.

Theorem 2.7. Let $E$ be a complete metric space and $F: E \rightarrow Z$ an open and minimal mapping of $E$ onto $Z$ for which $\operatorname{Dom} F$ is dense in $E$ and $F\left(x_{0}\right)=\bigcap_{i \geqslant 1} F\left(U_{i}\right)$ whenever $U_{i}, i=1,2,3, \ldots$, are open subsets of $E$ such that $\lim _{i \rightarrow \infty} \operatorname{diam} U_{i}=0$ and 
$\left\{x_{0}\right\}=\bigcap_{i \geqslant 1} U_{i}$. Then the following statements are equivalent:

(a) $Z$ contains some dense completely metrisable subset $Z_{1}$;

(b) There exists a dense $G_{\delta}$ subset $G \subset E$ such that $F$ is single-valued and usc at every point $x \in G$.

Proof: To show that (a) implies (b) we need the following lemma.

LEMMA 2.8. Let $\alpha^{\prime}=\left\{U^{\prime}\right\}$ be a disjoint family of nonempty open subsets of $E$ and let $\delta>0$. Then there is a system $\alpha^{\prime \prime}=\left\{U^{\prime \prime}\right\}$ of nonempty open sets $U^{\prime \prime} \subset E$ such that

(i) $\alpha^{\prime \prime}$ is a disjoint system which is inscribed in $\alpha^{\prime}$, meaning that for every $U^{\prime \prime} \in \alpha^{\prime \prime}$ there is (a necessarily unique) $U^{\prime} \in \alpha^{\prime}$ such that $U^{\prime \prime} \subset U^{\prime}$;

(ii) $\operatorname{diam} U^{\prime \prime}<\delta$ for every $U^{\prime \prime} \in \alpha^{\prime \prime}$;

(iii) for every $U^{\prime \prime} \in \alpha^{\prime \prime} \operatorname{diam}\left(F\left(U^{\prime \prime}\right) \cap Z_{1}\right)<\delta$ and $\operatorname{cl} F\left(U^{\prime \prime}\right) \subset F\left(U^{\prime}\right)$, where $U^{\prime}$ is the only element of $\alpha^{\prime}$ containing $U^{\prime \prime}$;

(iv) $\bigcup\left\{U^{\prime \prime}: U^{\prime \prime} \in \alpha^{\prime \prime}\right\}$ is dense in $\bigcup\left\{U^{\prime}: U^{\prime} \in \alpha^{\prime}\right\}$.

Proof of Lemma 2.8: Systems $\alpha^{\prime \prime}$ satisfying (i), (ii) and (iii) do exist. For instance, take some $U_{0}^{\prime} \in \alpha^{\prime} . F\left(U_{0}^{\prime}\right) \neq \emptyset$ is an open set. Take some $z_{0} \in F\left(U_{0}^{\prime}\right) \cap Z_{1}$ and some open $V \ni z_{0}$ such that $\bar{V} \subset F\left(U_{0}^{\prime}\right)$ and $\operatorname{diam}\left(V \cap Z_{1}\right)<\delta$. By minimality of $F$ we can find some open $U_{0}^{\prime \prime} \neq \emptyset, U_{0}^{\prime \prime} \subset U_{0}^{\prime}$, with $F\left(U_{0}^{\prime \prime}\right) \subset V$ and $\operatorname{diam} U_{0}^{\prime \prime}<\delta$. Hence $\operatorname{diam}\left(F\left(U_{0}^{\prime \prime}\right) \cap Z_{1}\right)<\delta$ and the one element family $\left\{U_{0}^{\prime \prime}\right\}$ satisfies (i), (ii) and (iii). Now consider a maximal system $\alpha^{\prime \prime}=\left\{U^{\prime \prime}\right\}$ satisfying (i), (ii), (iii).

Let us show that it satisfies (iv) as well. For a contradiction, suppose there exists $U_{0}^{\prime} \in \alpha^{\prime}$ and $x_{0} \in U_{0}^{\prime} \backslash \overline{\bigcup\left\{U^{\prime \prime}: U^{\prime \prime} \in \alpha^{\prime \prime}\right\}}$. Then, as before, we can find a nonempty open set $U_{0}^{\prime \prime} \subset U_{0}^{\prime} \backslash \overline{\bigcup\left\{U^{\prime \prime}: U^{\prime \prime} \in \alpha^{\prime \prime}\right\}}$ such that $\operatorname{diam}\left(F\left(U_{0}^{\prime \prime}\right) \cap Z_{1}\right)<\delta, \operatorname{diam} U_{0}^{\prime \prime}<\delta$, and $\overline{F\left(U_{0}^{\prime \prime}\right)} \subset F\left(U_{0}^{\prime}\right)$. The system $\alpha^{\prime \prime} \cup\left\{U_{0}^{\prime \prime}\right\}$ is strictly larger than $\alpha^{\prime \prime}$ and still satisfies the conditions (i), (ii), (iii). This contradiction completes the proof of the lemma.

Consider now the one element family $\alpha^{0}=\{E\}$. Apply Lemma 2.8 with $\alpha^{\prime}:=\alpha^{0}$ and $\delta=1$. Denote the resulting system $\alpha^{\prime \prime}$ by $\alpha^{1}$. Then apply Lemma 2.8 with $\alpha^{\prime}:=\alpha^{1}$ and $\delta=1 / 2$. This yields a system $\alpha^{\prime \prime}$ which we denote by $\alpha^{2}$. Proceeding in this manner we construct inductively a sequence $\left(\alpha^{n}\right)_{n \geqslant 0}$ of systems such that, for every $n \geqslant 1$,

(i) $\alpha^{n}$ is a disjoint family of nonempty open sets which is inscribed in $\alpha^{n-1}$;

(ii) $\operatorname{diam} U<1 / n$ for every $U \in \alpha^{n}$;

(iii) For every $U^{n} \in \alpha^{n}, \operatorname{diam}\left(F\left(U^{n}\right) \cap Z_{1}\right)<1 / n$ and $\overline{F\left(U^{n}\right)} \subset F\left(U^{n-1}\right)$, where $U^{n-1}$ is the element of $\alpha^{n-1}$ for which $U^{n} \subset U^{n-1}$;

(iv) $G^{n}:=\bigcup\left\{U^{n}: U^{n} \in \alpha^{n}\right\}$ is dense in $E$.

Put $G:=\bigcap_{n \geqslant 1} G^{n}$. Take $x_{0} \in G$. Then there is a uniquely determined sequence 
$\left(U^{n}\right)_{n \geqslant 1}$ of open sets $U^{n} \in \alpha^{n}$ such that $x_{0}=\bigcap_{n \geqslant 1} U^{n}$ and (iii) is satisfied. Since $Z_{1}$ is a complete metric space there is some $z_{0} \in Z_{1}$ such that $\left\{z_{0}\right\}=\bigcap_{i \geqslant 1}\left(\overline{F\left(U^{i}\right)} \cap Z_{1}\right)=$ $\bigcap_{i \geqslant 1}\left(F\left(U^{i}\right) \cap Z_{1}\right)$.

Let $W \ni z_{0}$ be some open set in $Z$. Find some open $V \subset Z$ such that $z_{0} \in V \subset$ $\bar{V} \subset W$. There is some integer $n>0$ for which $F\left(U^{n}\right) \cap Z_{1} \subset V$. Since $Z_{1}$ is dense in $Z$ we have $\overline{F\left(U^{n}\right)}=\overline{F\left(U^{n}\right) \cap Z_{1}} \subset \bar{V} \subset W$. This implies that $\left\{z_{0}\right\}=\bigcap_{i \geqslant 1} \overline{F\left(U_{i}\right)} \subset$ $\bigcap_{i \geqslant 1} F\left(U_{i}\right)=F\left(\bigcap_{i \geqslant 1} U_{i}\right)=F\left(x_{0}\right)$ and that $F$ is usc at $x_{0}$. The proof that (a) implies (b) is completed.

(b) $\Longrightarrow\left(\right.$ a). Let $\left(U_{n}^{*}\right)_{n \geqslant 1}$ be open and dense subsets of $E$ and let $F$ be singlevalued and usc at the points of $G=\bigcap_{n \geqslant 1} U_{n}^{*}$. We first show that $Z$ is a Baire space. That is, the intersection $\bigcap_{n \geqslant 1} H_{n}$ of any sequence $\left(H_{n}\right)_{n \geqslant 1}$ of open and dense sets is dense in $Z$. Let $V \neq \emptyset$ be an open subset of $Z$. Since $F: E \rightarrow Z$ is onto and $V \cap H_{1} \neq \emptyset$ the minimality of $F$ implies that for some nonempty open $U_{1} \subset E, U_{1} \subset U_{1}^{*}$, the open set $F\left(U_{1}\right) \subset V \cap H_{1}$. We can assume that $\operatorname{diam} U_{1}<1 / 2$. Further, since $F\left(U_{1}\right) \cap H_{2} \neq \emptyset$, the minimality of $F$ implies that we can find some non-empty open set $U_{2} \subset E$ such that $\operatorname{diam} U_{2}<1 / 2^{2}, \bar{U}_{2} \subset U_{1}, U_{2} \subset U_{2}^{*}$ and $F\left(U_{2}\right) \subset V \cap H_{2}$. Continuing this process we construct a sequence $\left\{U_{n}\right\}_{n \geqslant 1}$ of nonempty open subsets of $E$ such that, for every integer $n>0, \overline{U_{n+1}} \subset U_{n}, \operatorname{diam} U_{n}<1 / 2^{n}, U_{n} \subset U_{n}^{*}$ and $F\left(U_{n}\right) \subset H_{n} \cap V$. For the point $\left\{x_{0}\right\}=\bigcap_{n \geqslant 1} U_{n} \subset G$ we have $F\left(x_{0}\right)=\bigcap_{n \geqslant 1} F\left(U_{n}\right) \in\left(\bigcap_{n \geqslant 1} H_{n}\right) \cap V$. Thus the latter set is nonempty.

To show that $Z$ contains a dense completely metrisable set $Z_{1}$ we proceed as follows. Let $\alpha_{1}=\{U\}$ be a maximal system of nonempty open subsets of $E$ such that (1) $\operatorname{diam} U<1 / 2$ for all $U \in \alpha_{1}$; (2) $U \subset U_{1}^{*}$ for all $U \in \alpha_{1}$ and (3) $\left\{F(U): U \in \alpha_{1}\right\}$ is a disjoint family of open subsets of $Z$. Necessarily, $\alpha_{1}=\{U\}$ is also a disjoint system. We show now that the open set $H_{1}:=\bigcup\{F(U): U \in \alpha\}$ is dense in $Z$.

Suppose the contrary: $Z \backslash \overline{H_{1}} \neq \emptyset$. Since $F: E \rightarrow Z$ is onto, there exists some open $U^{\prime} \subset U_{1}^{*}, \operatorname{diam} U^{\prime}<1 / 2$ for which $F\left(U^{\prime}\right) \subset Z \backslash \overline{H_{1}}$. This contradicts maximality of $\alpha_{1}$ since the family $\alpha_{1} \cup\left\{U^{\prime}\right\}$ is bigger than $\alpha_{1}$ and still satisfies the conditions (1) - (3). Further we take a maximal system $\alpha_{2}=\{U\}$ which is strongly inscribed in $\alpha_{1}$ (the latter means that the closure of every element of $\alpha_{2}$ is contained into some element of $\alpha_{1}$ and is such that, for every $U \in \alpha_{2}:\left(1_{2}\right) \operatorname{diam} U<1 / 2^{2} ;\left(2_{2}\right) U \subset U_{2}^{*}$ and $\left(3_{2}\right)\left\{F(U): U \in \alpha_{2}\right\}$ is a disjoint family of open subsets of $Z$. Necessarily, $\alpha_{2}$ is also a disjoint system and every $U_{2} \in \alpha_{2}$ uniquely determines some $U_{1} \in \alpha_{1}$ so that $\overline{U_{2}} \subset U_{1}$. It is not difficult to see that the set $H_{2}:=\bigcup\left\{F(U): U \in \alpha_{2}\right\}$ is dense in $H_{1}$ 
and, therefore, in $Z$. Proceeding in this way we construct a sequence $\alpha_{n}$ of disjoint families of open sets such that, for every $U \in \alpha_{n}$,

(1n $) \operatorname{diam} U<1 / 2^{n}$

$\left(2_{n}\right) \quad U \subset U_{n}^{*}$

$\left(3_{n}\right) \quad H_{n}:=\bigcup\left\{F(U): U \in \alpha_{n}\right\}$ is open and dense in $Z$.

Since $Z$ is a Baire space, the set $Z_{1}:=\bigcap_{n \geqslant 1} H_{n}$ is dense in $Z$. Every point $z_{0} \in Z_{1}$ uniquely identifies a sequence $\left(U_{n}\right)_{n \geqslant 1}, U_{n} \in \alpha_{n}$, such that $z_{0} \in F\left(U_{n}\right)$ for $n=1,2, \ldots$ Properties $\left(1_{n}\right)-\left(3_{n}\right)$ show that $g\left(z_{0}\right):=\bigcap_{n \geqslant 1} U_{n}$ is a singleton which belongs to $G$. The single-valued map $g: Z_{1} \rightarrow G$ is continuous and $F\left(g\left(z_{0}\right)\right)=$ $F\left(\bigcap_{n \geqslant 1} U_{n}\right)=\bigcap_{n \geqslant 1} F\left(U_{n}\right) \ni z_{0}$. Since $F$ is single valued and usc at $g\left(z_{0}\right)$ we see that $F\left(g\left(z_{0}\right)\right)=z_{0}$ and that $g: Z_{1} \rightarrow G$ is a homeomorphism between $Z_{1}$ and the $G_{\delta}$ set $\bigcap_{n \geqslant 1}\left(\bigcup\left\{U: U \in \alpha_{n}\right\}\right)$ of the complete metric space $E$. It is known that in such a case $Z_{1}$ is completely metrisable.

\section{GENERIC SUP-WELL-POSEDNESS}

In this section we study the properties of the mapping $M: B(X \times Y) \rightarrow X$ defined by

$$
M(f):=\left\{x \in X: \inf _{y \in K x} f(x, y)=v_{f}\right\}
$$

We give conditions under which the restriction of $M$ on some subsets $E$ of $B(X \times Y)$ is a minimal or minimal and open mapping with dense domain. This opens the way to apply the general results from Section 2 in order to derive that $M$ is single - valued and usc at the points of some dense $G_{\delta}$ subset $G$ of $E$. Further, it turns out that for every $f \in G$ the problem $\left(P_{f}\right)$ is sup-well-posed.

Proposition 3.1. Let $\left\{f_{0}\right\}=\bigcap_{i \geqslant 1} B_{i}$ where $\left(B_{i}\right)_{i \geqslant 1}$ is a sequence of sets in $B(X \times Y)$ with $\lim _{i \rightarrow \infty} \operatorname{diam} B_{i}=0$. Then $M\left(f_{0}\right)=\bigcap_{i \geqslant 1} M\left(B_{i}\right)$.

Proof: Clearly, $M\left(f_{0}\right) \subset \bigcap_{i \geqslant 1} M\left(B_{i}\right)$. Let $x^{\prime} \notin M\left(f_{0}\right)$. Then there is some $x^{\prime \prime} \in X$ and some $\varepsilon>0$ such that $\inf _{y \in K x^{\prime \prime}} f_{0}\left(x^{\prime \prime}, y\right)>\inf _{y \in K x^{\prime}} f_{0}\left(x^{\prime}, y\right)+\varepsilon$.

Take the positive integer $n$ so large that $\operatorname{diam} B_{n}<\varepsilon / 2$. Then for each $f \in B_{n}$ we have $\left\|f-f_{0}\right\|<\varepsilon / 2$ and consequently $\inf _{y \in K x^{\prime \prime}} f\left(x^{\prime \prime}, y\right) \geqslant \inf _{y \in K x^{\prime \prime}} f_{0}\left(x^{\prime \prime}, y\right)-\varepsilon / 2>$ $\inf _{y \in K x^{\prime}} f_{0}\left(x^{\prime}, y\right)+\varepsilon / 2 \geqslant \inf _{y \in K x^{\prime}} f\left(x^{\prime}, y\right)$. This shows that $x^{\prime} \notin M(f)$ for every $f \in B_{n}$. Hence $x^{\prime} \notin M\left(B_{n}\right)$ and $x^{\prime} \notin \bigcap_{i \geqslant 1} M\left(B_{i}\right)$. 
Proposition 3.2. Let $E \subset B(X \times Y)$ be a subset such that $f(x, y)+g(x)$ belongs to $E$ whenever $g(x)$ is a continuous function on $X$. Then the restriction of $M$ on $E$ is a minimal mapping.

Proof: We shall denote the restriction of $M$ on $E$ by $M$ again. Let $U \subset E$ and $V \subset X$ be open sets and let $x_{0} \in M\left(f_{0}\right) \cap V$ for some $f_{0} \in U$. Let $g: X \longrightarrow[0,1]$ be a continuous function such that $g\left(x_{0}\right)=1$ and $g(X \backslash V)=0$. Let $\varepsilon>0$ be so small that $h:=f_{0}+\varepsilon g \in U$. For $y \in K x_{0}, h\left(x_{0}, y\right)=f_{0}\left(x_{0}, y\right)+\varepsilon$ and $\inf _{y \in K x_{0}} h\left(x_{0}, y\right)=$ $\inf _{y \in K x_{0}} f_{0}\left(x_{0}, y\right)+\varepsilon=v_{f_{0}}+\varepsilon$. On the other hand, for $x \notin V$ and $y \in K x$, we have $\inf _{y \in K x} h(x, y)=\inf _{y \in K x} f_{0}(x, y) \leqslant v_{f_{0}}$. Therefore $v_{h} \geqslant v_{f_{0}}+\varepsilon>v_{f_{0}} \geqslant \sup _{x \notin V} \inf _{y \in K x} h(x, y)$. It is easy to derive from here that for all functions $f$ from the open ball $B(h, \varepsilon / 2)$, $v_{f}>\sup _{\inf } f(x, y)$. This implies that $M(B(h, \varepsilon / 2)) \subset V$. Clearly, $h \in U_{0}:=$ $U \cap B(h, \varepsilon / 2)$ and $M\left(U_{0}\right) \subset V$.

Proposition 3.3. Let $E$ be a subset of $B(X \times Y)$ containing the constant functions and such that whenever $f, g \in E$, so do $f+g, \max \{f, g\}, \min \{f, g\}$. Then the domain Dom $M:=\{f \in B(X \times Y): M(f) \neq \emptyset\}$ is dense in $E$.

Proof: Let $f \in E$ and $\varepsilon>0$. Consider the function

$$
f_{\varepsilon}(x, y)=\min \left\{f(x, y), v_{f}-\varepsilon\right\}+\max \left\{f(x, y), v_{f}+\varepsilon\right\}-v_{f}
$$

Clearly, $f_{\varepsilon} \in E$. We shall show that $\left\|f-f_{\varepsilon}\right\| \leqslant \varepsilon$ and $M\left(f_{\varepsilon}\right) \neq \emptyset$. Take an arbitrary point $(x, y) \in X \times Y$. If $f(x, y)<v_{f}-\varepsilon$, then $f_{\varepsilon}(x, y)=f(x, y)+\left(v_{f}+\varepsilon\right)-v_{f}=$ $f(x, y)+\varepsilon$. Similarly, if $f(x, y)>v_{f}+\varepsilon$, then $f_{\varepsilon}(x, y)=f(x, y)-\varepsilon$. It remains to consider the case when $\left|f(x, y)-v_{f}\right| \leqslant \varepsilon$. In this case we have $f_{\varepsilon}(x, y)=\left(v_{f}-\varepsilon\right)+$ $\left(v_{f}+\varepsilon\right)-v_{f}=v_{f}$. Thus $\left\|f-f_{\varepsilon}\right\| \leqslant \varepsilon$. Now we shall show that $M\left(f_{e}\right) \neq \emptyset$. Indeed, there exists some $x^{*} \in X$ with $v_{f}+\varepsilon>v_{f} \geqslant \inf _{y \in K x^{*}} f\left(x^{*}, y\right)>v_{f}-\varepsilon$. There is some $y^{*} \in K x^{*}$ for which $v_{f}+\varepsilon>f\left(x^{*}, y^{*}\right) \geqslant \inf _{y \in K x^{*}} f\left(x^{*}, y\right)>v_{f}-\varepsilon$. Then $f_{\varepsilon}\left(x^{*}, y^{*}\right)=v_{f}$. Also, for every $y \in K x^{*}$ we have $f\left(x^{*}, y\right) \geqslant \inf _{y \in K x^{*}} f\left(x^{*}, y\right)>v_{f}-\varepsilon$ and hence $f_{e}\left(x^{*}, y\right)=\left(v_{f}-\varepsilon\right)+\max \left\{f\left(x^{*}, y\right), v_{f}+\varepsilon\right\}-v_{f} \geqslant\left(v_{f}-\varepsilon\right)+\left(v_{f}+\varepsilon\right)-v_{f}=v_{f}$. Thus $\inf _{y \in K x^{*}} f_{\varepsilon}\left(x^{*}, y\right)=v_{f}$.

Further, for every $x^{\prime} \in X$, we have $\inf _{y \in K x^{\prime}} f\left(x^{\prime}, y\right) \leqslant v_{f}<v_{f}+\varepsilon$. There exists some $y^{\prime} \in K x^{\prime}$ such that $f\left(x^{\prime}, y^{\prime}\right)<v_{f}+\varepsilon$. Then $f_{\varepsilon}\left(x^{\prime}, y^{\prime}\right)=\min \left\{f\left(x^{\prime}, y^{\prime}\right), v_{f}-\varepsilon\right\}+$ $\left(v_{f}+\varepsilon\right)-v_{f} \leqslant\left(v_{f}-\varepsilon\right)+\left(v_{f}+\varepsilon\right)-v_{f}=v_{f}$. This implies that $\inf _{y \in K x^{\prime}} f_{\varepsilon}\left(x^{\prime}, y\right) \leqslant v_{f}$. We get that $x^{*} \in M\left(f_{e}\right)$.

Bearing in mind our needs in Section 4 we note here that $\left(x^{*}, y^{*}\right)$ is actually a solution of $\left(P_{f_{\varepsilon}}\right)$, that is, $\left(x^{*}, y^{*}\right) \in S\left(f_{\varepsilon}\right)$. In particular, it follows from Proposition 3.3. 
that Dom $S$ is dense in $E$ whenever the requirements of that proposition are satisfied. More generally, it was proved above that every pair $\left(x^{*}, y^{*}\right) \in X \times Y$ for which $y^{*} \in K x^{*}$ and $v_{f}+\varepsilon>f\left(x^{*}, y^{*}\right) \geqslant \inf _{y \in K x^{*}} f\left(x^{*}, y\right)>v_{f}-\varepsilon$, is a solution of $\left(P_{f_{\varepsilon}}\right)$.

Proposition 3.4. Suppose $E \subset B(X \times Y)$ satisfies the requirements of Proposition 3.3 and, in addition, satisfies that for every $f \in E, \inf _{y \in K x} f(x, y)$ is a lsc function of $x$. Then the restriction of $M$ on $E$ is an open mapping.

Proof: Let $x_{0} \in M(f)$ and $\varepsilon>0$. With $f_{\varepsilon}$ as in the proof of Proposition 3.3, it suffices to show that $M\left(f_{\varepsilon}\right)$ contains some neighbourhood of $x_{0}$. Since $\inf _{y \in K x_{0}} f\left(x_{0}, y\right)=v_{f_{0}}$ and $\inf _{y \in K x} f(x, y)$ is Isc at $x_{0}$, there exists some open $V \ni x_{0}$ such that $\inf _{y \in K_{x}} f(x, y)>v_{f}-\varepsilon$ for every $x \in V$. This means that for $x \in V$ and $y \in K x$ we have $f(x, y) \geqslant \inf _{y \in K x} f(x, y)>v_{f}-\varepsilon$. Therefore,

$$
f_{\varepsilon}(x, y)=\left(v_{f}-\varepsilon\right)+\max \left\{f(x, y), v_{f}+\varepsilon\right\}-v_{f} \geqslant v_{f} \text { and } \inf _{y \in K_{x}} f_{\varepsilon}(x, y) \geqslant v_{f}
$$

On the other hand, as we have seen in the proof of Proposition 3.3.,

$$
\inf _{y \in K x^{\prime}} f_{\varepsilon}\left(x^{\prime}, y\right) \leqslant v_{f} \text { for every } x^{\prime} \in X
$$

Hence we have that $M\left(f_{\varepsilon}\right) \supset V$.

In the sequel we shall need also the following observation.

LEmma 3.5. For every $f \in B(X \times Y)$ and $\varepsilon>0$ the following inequality holds:

$$
\sup _{x \notin M\left(f_{e}\right)} \inf _{y \in K x} f(x, y) \leqslant v_{f}-\varepsilon<v_{f}
$$

Proof: In the proof of Proposition 3.3 we established that if $x^{*} \in X$ is such that $\inf _{y \in K x^{*}} f\left(x^{*}, y\right)>v_{f}-\varepsilon$, then $x^{*} \in M\left(f_{\varepsilon}\right)$.

The connection between the properties of $M$ and the sup-well-posedness is described in the next statement.

Proposition 3.6. Let $E$ be as in Proposition 3.3 and $f \in E$. Then the following conditions are equivalent:

(a) $M: E \rightarrow X$ is single - valued and usc at $f$;

(b) $\left(P_{f}\right)$ is sup-well posed.

Proof: Let $M(f)=\left\{x_{0}\right\}$ and let $M$ be usc at $f$. Take some open $V \ni x_{0}$ and let $\left(x_{i}\right)_{i \geqslant 1}$ be a maximising sequence for $\left(P_{f}\right)$, that is, $\lim _{i \rightarrow \infty} \inf _{y \in K x_{i}} f\left(x_{i}, y\right)=v_{f}$. 
Since $M$ is usc at $f$, there exists some $\varepsilon>0$ such that $M\left(f_{\varepsilon}\right) \subset V$. By Lemma 3.5 , the sequence $\left(x_{i}\right)_{i \geqslant 1}$ eventually belongs to $V$. This shows that $\lim _{x_{i} \rightarrow \infty} x_{i}=x_{0}$ and that $(\mathrm{a}) \Rightarrow(\mathrm{b})$. Suppose now that $\left(P_{f}\right)$ is sup-well-posed and has sup-solution $x_{0}=M(f)$ but $M$ is not usc at $f$. Then there exists some open $V \ni x_{0}$ and a sequence $\left(f_{n}\right)_{n \geqslant 1} \subset E$ such that $\left\|f_{n}-f\right\| \rightarrow 0$ but $M\left(f_{n}\right) \backslash V \neq \emptyset$ for every $n \geqslant 1$. But for every sequence $\left(x_{n}\right)_{n \geqslant 1}$ with $x_{n} \in M\left(f_{n}\right)$ we have $v_{f_{n}}=\inf _{y \in K x_{n}} f_{n}\left(x_{n}, y\right)$. Since $\left\|f_{n}-f\right\| \rightarrow 0,\left|v_{f_{n}}-v_{f}\right| \rightarrow 0$ and therefore $\left(x_{n}\right)_{n \geqslant 1}$ is a maximising sequence for $\left(P_{f}\right)$ and must converge to $x_{0}$. This is a contradiction.

THEOREM 3.7. Let $E$ be a closed subset of $(B(X \times Y),\|\cdot\|)$ which satisfies the requirements:

(1) E contains all bounded continuous functions $g(x)$ defined in $X$ (each such function $g$ is naturally identified with some function $\tilde{g}$ in $B(X \times Y)$ by $\tilde{g}(x, y):=g(x)$ for $(x, y) \in X \times Y)$;

(2) for every pair $f_{1}, f_{2} \in E$ the functions $f_{1}+f_{2}, \max \left\{f_{1}, f_{2}\right\}, \min \left\{f_{1}, f_{2}\right\}$ also belong to $E$;

(3) for every $f \in E$ the function $\inf _{y \in K x} f(x, y)$ is lsc in $X$.

Then the following statements are equivalent

(a) $X$ contains a dense completely metrisable subset;

(b) $E$ is generically sup-well posed.

Proof: Consider the mapping $M: E \rightarrow X$. Since $E$ contains the function $f_{0}(x, y) \equiv 1$ and $M\left(f_{0}\right)=X$, this mapping is "onto". Propositions 3.1. through 3.4 indicate that Theorem 2.7 can be applied with $F \equiv M$ and $Z \equiv X$. The rest of the proof is contained in Proposition 3.6.

Condition (3) from Theorem 3.7 is connected with the properties of the mapping $K$. Closely related to $K$ is the mapping $K^{\prime}: X \rightarrow G r K$ which is defined by the formula $K^{\prime} \boldsymbol{x}=\{\boldsymbol{x}\} \times K \boldsymbol{x}$ and maps $X$ into the graph of $K, \operatorname{Gr} K:=\{(\boldsymbol{x}, y) \in X \times Y: y \in K x\}$. $K$ is called graph usc if $K^{\prime}$ is usc. If $K$ is graph usc, then it is usc. The two notions coincide if $K$ is usc and $K x$ is a nonempty compact subset of $Y$ for every $x \in X$ (in this case $K$ is called an usco mapping). The following lemma is standard in optimisation: we omit the simple proof.

LEMma 3.8. If $K$ is graph usc and $f$ is a bounded lsc function in $X \times Y$, then $\inf _{y \in K x} f(x, y)$ is a Isc function of $x$.

Corollary 3.9. Let $K: X \rightarrow Y$ be graph usc and let $E$ be the space $C(X \times Y)(L(X \times Y))$ of all bounded continuous (lower semicontinuous) real-valued functions. Then the statements (a) and (b) from Theorem 3.7 are equivalent. In particular, this happens if $K$ is an usco mapping. 
In some cases we can prove that $E$ is generically sup-WP without requiring usc of $K$ or $K^{\prime}$. In these cases we can not apply Theorem 2.7 because the mapping $M$ is not, in general, open.

The mapping $K: X \rightarrow Y$ is called lower semicontinuous (lsc) at $x_{0} \in X$ if for every open $W \subset Y$ such that $W \cap K x_{0} \neq \emptyset$, there exists some open $V \ni x_{0}$ such that $x \in V$ implies $K x \cap W \neq \emptyset . K$ is called $l s c$ if it is lsc at every $x \in X$.

THEOREM 3.10. Let $(X, \tau)$ be a topological space which is fragmented by some complete metric $d(\cdot, \cdot)$ such that the identity map $(X, d) \longleftrightarrow(X, \tau)$ is continuous. Let $K: X \rightarrow Y$ be lsc. Then the set $E=C(X \times Y)$ of all continuous functions in $(X, \tau) \times Y$ is generically sup-WP. In particular, this is true if $(X, \tau)$ is a complete metric space.

Proof: We apply Theorem 2.4 and find a dense $G_{\delta}$ subset $G \subset E$ such that for every $f_{0} \in G$ there is a point $x_{0} \in X$ and a sequence $\left(U_{n}\right)_{n \geqslant 1}$ of open sets in $E$ with $f_{0}=\bigcap_{n \geqslant 1} U_{n},\left\{x_{0}\right\}=\bigcap_{n \geqslant 1} \overline{M\left(U_{n}\right)}{ }^{d}$ and $\operatorname{diam} M\left(U_{n}\right) \leqslant 1 / n$. Note that $M\left(f_{0}\right)=\bigcap_{n \geqslant 1} M\left(U_{n}\right) \subset \bigcap_{n \geqslant 1} \overline{M\left(U_{n}\right)}{ }^{d}=\left\{x_{0}\right\}$. We shall show that $\left(P_{f_{0}}\right)$ is sup-WP and $M\left(f_{0}\right)=\left\{x_{0}\right\}$.

Let $\left(x_{i}\right)_{i \geqslant 1}$ be a maximising sequence for $\left(P_{f_{0}}\right)$. That is, $\inf _{y \in K x_{i}} f_{0}\left(x_{i}, y\right) \rightarrow v_{f_{0}}$. By Lemma 3.5 the set $M\left(U_{n}\right)$ eventually contains the sequence $\left(x_{i}\right)_{i \geqslant 1}$. Therefore $\left(x_{i}\right)_{i \geqslant 1}$ is a Cauchy sequence which can converge in $d(\cdot, \cdot)$ only to $x_{0}$ (and then converges also to $x_{0}$ in $\tau$ ). Let $y_{0} \in K x_{0}$ and $\varepsilon>0$. Find some open sets $V \ni x_{0}$ and $W \ni y_{0}$ such that $f_{0}(V \times W)<f_{0}\left(x_{0}, y_{0}\right)+\varepsilon$. Without loss of generality we can suppose that $K x \cap W \neq \emptyset$ for every $x \in V$, because $K$ is lsc at $x_{0}$. For $x_{i} \in V$ we have some $y_{i} \in K x_{i} \cap W$. For such $i$ we have $f_{0}\left(x_{0}, y_{0}\right)+\varepsilon>f_{0}\left(x_{i}, y_{i}\right) \geqslant \inf _{y \in K x_{i}} f_{0}\left(x_{i}, y\right)$. Passing to limits as $i \rightarrow \infty$ we get $f_{0}\left(x_{0}, y_{0}\right)+\varepsilon \geqslant v_{f_{0}}$. It follows that $\inf _{y \in K x_{i}} f_{0}\left(x_{0}, y\right) \geqslant v_{f_{0}}$. This means that $x_{0} \in M\left(f_{0}\right)$.

Corollary 3.11. Let $X$ be a complete metric space and let $E=C(X \times Y)$ be the space of all continuous bounded functions in $X \times Y$. Then there exists a dense $G_{\delta}$ subset $G \subset E$ such that the problem

$$
\sup _{X} \inf _{Y} f(x, y)
$$

is sup-well-posed for every $f \in G$.

Proof: Put $K x:=Y$ for every $x \in X$ and apply Theorem 3.10.

Corollary 3.12. Let $X$ be a topological space and $E$ be either the space $L(X)$ of all bounded lsc functions in $X$ or the space $C(X)$ of all bounded continuous 
functions in $X$ (in both cases with the norm $\|f\|=\sup _{X}|f(x)|$ ). Then the following statements are equivalent:

(a) $X$ contains a dense completely metrisable subset;

(b) $E$ is generically Tykhonov well-posed.

Proof: Let $Y=\left\{y_{0}\right\}$ be a one point set with the discrete topology. The space $L(X \times Y)(C(X \times Y))$ is naturally identifiable with $L(X)(C(X))$. The function $f(x, y)$ is sup-WP if, and only if, the corresponding function $\tilde{f}(x) \equiv f\left(x, y_{0}\right)$ is Tykhonov well posed. The claim follows from Corollary 3.9.

The equivalence of (a) and (b) for the case $E=C(X)$ was established in [4] and [5] for compact spaces $X$ and in [6] for arbitrary topological space $X$.

\section{Generic Well posedness}

Using the results from the previous sections we give here sufficient conditions under which $L(X \times Y)$ (or $C(X \times Y)$ ) is generically well posed.

Lemma 4.1. Let $Y$ be a complete metric space and $K: X \longrightarrow Y$ usco. Let $\varepsilon>0$ and $f: X \times Y \longrightarrow R$ a bounded lsc function. Then for every $\delta>0$ there exist some open sets $V \subset X, W \subset Y$ such that

(1) $V \subset V_{0}:=\left\{x \in X: \inf _{y \in K x} f(x, y)>v_{f}-\varepsilon\right\}$,

(2) $\operatorname{diam} W<\delta$,

(3) for every $x \in V$ there exists some $y_{x} \in K x \cap \bar{W}$ with $f\left(x, y_{x}\right) \leqslant v_{f}+\varepsilon$. In particular, for every $x \in V$ we have

$$
v_{f}-\varepsilon<\inf _{y \in K \boldsymbol{x}} f(x, y) \leqslant \inf _{y \in K \boldsymbol{x} \cap \bar{W}} f(x, y) \leqslant v_{f}+\varepsilon .
$$

Proof: Since $\inf _{y \in K x} f(x, y)$ is lsc (because $K$ is usco), the set $V_{0}$ is open and nonempty. Take some $x_{0} \in V_{0}$.

Let $W_{1}, W_{2}, \ldots, W_{k}$ be a finite cover of $K x_{o}$ consisting of open sets of diameter less than $\delta$. There exists some open $V_{1} \ni x_{0}, V_{1} \subset V_{0}$, such that $K x \subset \bigcup_{i=1}^{k} W_{i}$ whenever $x \in V_{1}$ (because $K$ is usc at $x_{0}$ ). If for every $x \in V_{1}$ there is some $y_{x} \in K x \cap \bar{W}_{1}$ with $f\left(x, y_{x}\right) \leqslant v_{f}+\varepsilon$, the proof is complete. If this is not the case, then there is some $x_{1} \in V_{1}$ for which either (i) $K x_{1} \cap \bar{W}_{1}=\emptyset$ or (ii) $\inf _{y \in K x_{1} \cap \bar{W}_{1} \neq \emptyset} f\left(x_{1}, y\right)>v_{f}+\varepsilon$. In the case (i) there exists some open $V_{2} \ni x_{1}, V_{2} \subset V_{1}$, such that $K x \cap \bar{W}_{1}=\emptyset$ whenever $x \in V_{2}$ (by the usco property of $K$ ). In case (ii) we can find some open $V_{2} \ni x_{1}, V_{2} \subset V_{1}$, so that $\inf _{y \in K x \bar{W}_{1}} f(x, y)>v_{f}+\varepsilon$ for every $x \in V_{2}$ (by the lsc property of $f$ and because $x \rightarrow K x \cap \bar{W}_{1}$ is an usco mapping). 
If the property (3) is satisfied with $V:=V_{2}$ and $W:=W_{2}$, we are done. If not, two alternatives arise again. Either there is some $x_{3} \in V_{2}$ for which $K x_{3} \cap \bar{W}_{2}=\emptyset$ or $K x_{3} \cap \bar{W}_{2} \neq \varnothing$ but $\inf _{y \in K x_{3} \cap W_{2}} f\left(x_{3}, y\right)>v_{f}+\varepsilon$. In both cases there is some open $V_{3} \ni x_{3}, V_{3} \subset V_{2}$, such that $\inf _{y \in K x \cap \bar{W}_{2}} f(x, y)>v_{f}+\varepsilon$ (if $K x \cap \bar{W}_{2}=\emptyset$ the corresponding "inf" is $+\infty$ by definition).

If (3) is satisfied with $V:=V_{3}$ and $W:=W_{3}$, the proof is over. If not, we can find some $V_{4} \subset V_{3}$ with $\inf _{y \in K x \cap \bar{W}_{3}} f(x, y)>v_{f}+\varepsilon$ for every $x \in V_{4}$. Proceeding in this way, at every step we either finish the proof or find another open set $V$ with the corresponding property. Finally we get a sequence $V_{0} \supset V_{1} \supset V_{2} \supset \cdots \supset V_{k}$ of nonempty open sets such that $\inf _{y \in K x \cap \bar{W}_{i-1}} f(x, y)>v_{f}+\varepsilon$ for every $x \in V_{i}, i=1,2 \ldots, k$. Then we must have (3) satisfied for $V:=V_{k}$ and $W=W_{k}$ because, otherwise, we would find some $x_{k} \in V_{k}$ with $\inf _{y \in K_{x_{k}} \cap \bar{W}_{i}} f\left(x_{k}, y\right)>v_{f}+\varepsilon$ for every $i=1,2, \ldots, k$. Then we get the contradiction:

$$
v_{f} \geqslant \inf _{y \in K x_{k}} f\left(x_{k}, y\right)=\min \left\{\inf _{y \in K x_{k} \cap \bar{W}_{i}} f\left(x_{k}, y\right): i=1,2, \ldots, k\right\}>v_{f}+\varepsilon
$$

Lemma 4.2. Let $X$ and $Y$ be topological spaces. Let $f \in L(X \times Y), \varepsilon>0$ and let the open sets $V \subset X, W \subset Y$ be such that, for all $x \in V, v_{f}-\varepsilon<\inf _{y \in K x} f(x, y) \leqslant$ $\inf _{y \in K x \cap \bar{W}} f(x, y)<v_{f}+\varepsilon$. Then for every continuous function $h: Y \longrightarrow[0,1]$ with $h(\bar{W})=0$ and for every open set $W^{\prime}$ with $h\left(Y \backslash W^{\prime}\right)=1$ there is some $f^{\prime} \in L(X \times Y)$, $\left\|f^{\prime}-f\right\|<10 \varepsilon$, such that $S\left(h^{\prime}\right) \subset V \times W^{\prime}$ whenever $\left\|h^{\prime}-f^{\prime}\right\|<\varepsilon / 2$.

PROOF: Let $x_{0} \in V$ and $g: X \rightarrow[-1,0]$ be a continuous function such that $g\left(x_{0}\right)=0$ and $g(X \backslash V)=-1$. Put $f^{\prime}(x, y):=f(x, y)+6 \varepsilon g(x)+3 \varepsilon h(y)$. Clearly, $\left\|f^{\prime}-f\right\| \leqslant 9 \varepsilon<10 \varepsilon$.

If $x \notin V$, we have $f^{\prime}(x, y)=f(x, y)-6 \varepsilon+3 \varepsilon h(y) \leqslant f(x, y)-3 \varepsilon$ because $h(y) \leqslant 1$. Consequently, for $x \notin V$, $\inf _{y \in K x} f^{\prime}(x, y) \leqslant \inf _{y \in K x} f(x, y)-3 \varepsilon \leqslant v_{f}-3 \varepsilon$. On the other hand, for $x=x_{0}$ and $y \in K x_{0}$ we get $f^{\prime}\left(x_{0}, y\right)=f\left(x_{0}, y\right)+3 \varepsilon h(y) \geqslant$ $\inf _{y \in K x_{0}} f\left(x_{0}, y\right)>v_{f}-\varepsilon$. Then $\inf _{y \in K x_{0}} f^{\prime}\left(x_{0}, y\right) \geqslant v_{f}-\varepsilon$. Hence $v_{f^{\prime}}=\sup _{X} \inf _{y \in K x} f^{\prime}(x, y) \geqslant$ $\inf _{y \in K x_{0}} f^{\prime}\left(x_{0}, y\right) \geqslant v_{f}-\varepsilon>v_{f}-3 \varepsilon \geqslant \sup _{x \notin V} \inf _{y \in K x} f^{\prime}(x, y)$. This shows that, for every function $h^{\prime}(x, y)$ with $\left\|h^{\prime}-f^{\prime}\right\|<\varepsilon$, the inequality $v_{h^{\prime}}>\sup \inf h^{\prime}(x, y)$ holds. In turn, this inequality implies that, if $\left(x^{\prime}, y^{\prime}\right) \in S\left(h^{\prime}\right)$, then $x^{\prime} \in V$. Now we shall show that the second coordinate $y^{\prime}$ belongs to $W^{\prime}$ if $\left\|h^{\prime}-f^{\prime}\right\|<\varepsilon / 2$. If $x \in V$ and $y \in K x \backslash W^{\prime}$ we have $h(y)=1$ and therefore $f^{\prime}(x, y)=f(x, y)+6 \varepsilon g(x)+3 \varepsilon \geqslant$ 
$\inf _{y \in K x} f(x, y)+6 \varepsilon g(x)+3 \varepsilon>\left(v_{f}-\varepsilon\right)+6 \varepsilon g(x)+3 \varepsilon=v_{f}+6 \varepsilon g(x)+2 \varepsilon$. For every $x \in V$ there is some $y_{x} \in K x \cap \bar{W}$ such that $f\left(x, y_{x}\right)<v_{f}+\varepsilon$. Then $f^{\prime}\left(x, y_{x}\right)=f\left(x, y_{x}\right)+6 \varepsilon g(x)<\left(v_{f}+\varepsilon\right)+6 \varepsilon g(x)$. Taken together all this means that, for $x \in V, \inf _{y \in K x \backslash W^{\prime}} f^{\prime}(x, y) \geqslant v_{f}+6 \varepsilon g(x)+2 \varepsilon>v_{f}+6 \varepsilon g(x)+\varepsilon \geqslant f^{\prime}\left(x, y_{x}\right) \geqslant \inf _{y \in K x} f^{\prime}(x, y)$. This implies that for all functions $h^{\prime}(x, y)$ with $\left\|h^{\prime}-f^{\prime}\right\|<\varepsilon / 2$ and $x \in V$ we have: $\inf _{y \in K x \backslash W^{\prime}} h^{\prime}(x, y)>\inf _{y \in K x} h^{\prime}(x, y)$. In particular, this inequality shows that, if $\left(x^{\prime}, y^{\prime}\right) \in S\left(h^{\prime}\right)$ and $x^{\prime} \in V$, then $y^{\prime} \in W^{\prime}$. Finally we get that $S\left(h^{\prime}\right) \subset V \times W^{\prime}$ if $\left\|h^{\prime}-f^{\prime}\right\|<\varepsilon / 2$.

THEOREM 4.3. Let $X$ contain a dense completely metrisable subset $X_{1}$ and let $Y$ be a complete metric space. Let $K: X \longrightarrow Y$ be an usco map and let $E=L(X \times Y)$ (or $E=C(X \times Y)$ ). Then $E$ is generically well posed.

Proof: First note that by Corollary 3.9 there is some dense $G_{\delta}$ subset $H \subset E$ such that $\left(P_{f}\right)$ is sup-WP whenever $f \in H$.

Further, put $U_{n}=\bigcup\left\{U: U\right.$ open in $E$ and $\left.\operatorname{diam}\left(S(U) \cap\left(X_{1} \times Y\right)\right)<1 / n\right\}$. We shall show that $U_{n}$ in dense in $E$. Take some open $U^{\prime} \neq \emptyset$ and some $f \in U^{\prime}$. Find $\varepsilon>0$ so that the ball $B(f, 11 \varepsilon) \subset U^{\prime}$. By Lemma 4.1 we can find some open sets $V \subset X$ and $W \subset Y$ such that properties (1), (2) and (3) from that lemma are satisfied. Without loss of generality we can consider that $\operatorname{diam}\left(V \cap X_{1}\right)<1 / n$ and $\operatorname{diam} \bar{W}<1 / n$. Take some open $W^{\prime} \supset \bar{W}$ with $\operatorname{diam} W^{\prime}<1 / n$ and apply Lemma 4.2. We get that $S\left(B\left(f^{\prime}, \varepsilon / 2\right)\right) \subset V \times W^{\prime}$. This shows that $U_{n} \cap U^{\prime} \supset B\left(f^{\prime}, \varepsilon / 2\right) \neq \emptyset$ which means that $U_{n}$ is dense in $E$. Put $G:=\bigcap_{u \geqslant 1} U_{n}$ and take some $f \in G \cap H$.

There are sequences $\left(U^{i}\right),\left(V^{i}\right),\left(W^{i}\right)$ of open subsets of $E, X$ and $Y$ respectively such that $S\left(U^{i}\right) \subset V^{i} \times W^{i}$ and $\operatorname{diam} W^{i}<1 / i$ for every $i \geqslant 1$. Let $\left(x_{p}, y_{p}\right)_{p \geqslant 1}$ be an optimising sequence for $\left(P_{f}\right)$, that is, $y_{i} \in K x_{i}, \inf _{y \in K x_{p}} f\left(x_{p}, y\right) \longrightarrow v_{f}$ and $f\left(x_{p}, y_{p}\right) \longrightarrow v_{f}$. Since $P(f)$ is sup-WP (because $\left.f \in H\right)\left(x_{p}\right)_{p \geqslant 1}$ converges to some $\boldsymbol{x}^{*} \in X$ such that $v_{f}=\inf _{y \in K_{x^{*}}} f\left(\boldsymbol{x}^{*}, y\right)$. Since $U^{n}$ is open, there is some $\delta>0$ for which the function $f_{\delta}(x, y)=\min \left\{f(x, y), v_{f}-\delta\right\}+\max \left\{f(x, y), v_{f}-\delta\right\}-v_{f}$ belongs to $U^{n}$. There exists $i_{0}$ such that, for $i \geqslant i_{0}, f\left(x_{i}, y_{i}\right)<v_{f}+\delta$ and $\inf _{y \in K_{x_{i}}} f\left(x_{i}, y\right)>v_{f}-\delta$. Then $\left\{\left(x_{i}, y_{i}\right): i \geqslant i_{0}\right\} \subset S\left(f_{\delta}\right) \subset S\left(U^{n}\right) \subset V^{n} \times W^{n}$. (See the remark after the proof of Proposition 3.3.) In particular, $\left\{y_{i}: i \geqslant i_{0}\right\} \subset W^{n}$. This shows that $\left(y_{i}\right)_{i \geqslant 1}$ is a Cauchy sequence in the complete metric space $Y$. Let $y^{*}=\lim _{i \rightarrow \infty} y_{i}$. Since the graph of the mapping $K$ is closed and $\left(x_{i}, y_{i}\right) \in G r K, y^{*} \in K x^{*}$. It follows by lower semicontinuity of $f$ that $f\left(\boldsymbol{x}^{*}, y^{*}\right) \leqslant \lim _{i \rightarrow \infty} f\left(x_{i}, y_{i}\right)=v_{f}$. Since $f\left(x^{*}, y^{*}\right) \geqslant \inf _{y \in K x^{*}} f\left(x^{*}, y\right)=v_{f}$ we see that $\left(x^{*}, y^{*}\right) \in S(f)$ and that $\left(P_{f}\right)$ is well posed. 
With stronger assumptions about the mapping $K$ we can prove generic well posedness under less restrictive conditions on the space $Y$.

Theorem 4.4. Let $K: X \longrightarrow Y$ be both usco and lsc. Then the following statements are equivalent

(a) $\operatorname{Gr} K:=\{(x, y) \in X \times Y: y \in K x\}$ contains a dense completely metrisable subset;

(b) $C(X \times Y)$ is generically well posed.

It is not difficult to guess that in the proof of this theorem we are going to apply Theorem 2.7. To be able to do so we need to investigate the properties of the mapping $S$. This is done in the following propositions that are also of independent interest. After that we give the proof of the theorem.

PRoposition 4.5. Let $X$ and $Y$ be topological spaces and let $K: X \longrightarrow Y$ be usco and lsc. Then the mapping $S: C(X \times Y) \rightarrow G r K$ is minimal.

Proof: Let $U^{\prime}, V^{\prime}, W^{\prime}$ be open subsets of $E=C(X \times Y), X$ and $Y$ respectively such that $\left(V^{\prime} \times W^{\prime}\right) \cap S\left(U^{\prime}\right) \neq \emptyset$. Take $\left(x_{0}, y_{0}\right), f \in U^{\prime}$ so that $\left(x_{0}, y_{0}\right) \in S(f) \cap\left(V^{\prime} \times W^{\prime}\right)$. Let $\varepsilon>0$ be such that $B(f, 11 \varepsilon) \subset U^{\prime}$. Find some open $W \ni y_{0}$ for which $\bar{W} \subset W^{\prime}$ and there is a continuous function $h: Y \rightarrow[0,1]$ with $h(W)=0$ and $h\left(Y \backslash W^{\prime}\right)=1$. By lower semicontinuity of $K$ we can assume that $K x \cap W \neq \emptyset$ for all $x$ from some open $V \ni x_{0}$. Without loss of generality we can assume that $\sup _{V \times W} f<v_{f}+\varepsilon$ and $V \subset V^{\prime}$. Moreover, we can suppose also that $\inf _{y \in K_{x}} f(x, y)>v_{f}-\varepsilon$ for all $x \in V$. It follows from Lemma 4.2 that there is some $f^{\prime}$, $\left\|f^{\prime}-f\right\|<10 \varepsilon$, such that $S\left(B\left(f^{\prime}, \varepsilon / 2\right)\right) \subset V \times W^{\prime} \subset V^{\prime} \times W^{\prime}$, where $B\left(f^{\prime}, \varepsilon / 2\right)$ is the open ball around $f^{\prime}$ of radius $\varepsilon / 2$. Since $B\left(f^{\prime}, \varepsilon / 2\right) \subset U^{\prime}$ we see that $S$ is a minimal mapping.

Proposition 4.6. Let $X$ and $Y$ be topological spaces and let $K: X \longrightarrow Y$ be usco and lsc. Then $S$ is an open mapping of $E=C(X \times Y)$ onto $Z=G r K$.

Proof: Let $\left(x_{0}, y_{0}\right) \in S(f)$ and let $\varepsilon>0$. Consider the function $f_{\varepsilon}$ of Proposition 3.3. It suffices to show that $S\left(f_{\varepsilon}\right)$ is a neighbourhood of $\left(x_{0}, y_{0}\right)$ in $Z$. We already know that there are some open sets $V \ni x_{0}$ and $W \ni y_{0}$ such that $\inf _{y \in K x} f(x, y)>v_{f}-\varepsilon$ for every $x \in V$ and $\sup _{V \times W} f<v_{f}+\varepsilon$. From the remark after the proof of Proposition 3.3 we know that $(V \times W) \cap G r K \subset S\left(f_{\varepsilon}\right)$.

ProposITION 4.7. Let $f_{0}=\bigcap_{i \geqslant 1} B_{i}$ where $\left(B_{i}\right)_{i \geqslant 1}$ is a sequence of sets in $L(X \times Y)$ with diam $B_{i} \longrightarrow 0$. Then $S\left(f_{0}\right)=\bigcap_{i \geqslant 1} S\left(B_{i}\right)$. 

ities:

Proof: Clearly, $S\left(f_{0}\right) \subset \bigcap_{i \geqslant 1} S\left(B_{i}\right)$. Let $\left(x^{\prime}, y^{\prime}\right) \notin S\left(f_{0}\right)$. There are two possibil(a) $\inf _{y \in K x^{\prime}} f_{0}\left(x^{\prime}, y\right)<v_{f}$, or

(b) $\inf _{y \in K x^{\prime}} f_{0}\left(x^{\prime}, y\right)=v_{f}<f_{0}\left(x^{\prime}, y^{\prime}\right)$.

In case (a) there is some $x^{\prime \prime} \in X$ and some $\varepsilon>0$ so that

$$
\inf _{y \in K x^{\prime}} f_{0}\left(x^{\prime}, y\right)<\inf _{y \in K x^{\prime \prime}} f_{0}\left(x^{\prime \prime}, y\right)-3 \varepsilon
$$

In case (b) there is some $y^{\prime \prime} \in K x^{\prime}$ and some $\varepsilon>0$ so that

$$
f_{0}\left(x^{\prime}, y^{\prime}\right)-3 \varepsilon>f_{0}\left(x^{\prime}, y^{\prime \prime}\right) \text {. }
$$

Then for any function $h,\left\|h-f_{0}\right\|<\varepsilon$, we have: in case (a) $\inf _{y \in K x^{\prime}} h\left(x^{\prime}, y\right)<$ $\inf _{y \in K x^{\prime \prime}} h\left(x^{\prime \prime}, y\right) \leqslant v_{h}$, and in case (b) $h\left(x^{\prime}, y^{\prime}\right)>h\left(x^{\prime}, y^{\prime \prime}\right)$.

In both cases $\left(x^{\prime}, y^{\prime}\right) \notin S(h)$. Take the integer $i_{0}$ so large that $\operatorname{diam} B_{i_{0}}<\varepsilon$. Then $\left\|f_{0}-h\right\|<\varepsilon$ for every $h \in B_{i_{o}}$ and $\left(x^{\prime}, y^{\prime}\right) \notin S\left(B_{i_{o}}\right)$.

Proposition 4.8. The problem $\left(P_{f}\right)$ is well posed if, and only if, $S$ is singlevalued and usc at $f$.

Proof: Let $\left(P_{f}\right)$ be well posed and $\left(x_{0}, y_{0}\right)=S(f)$. Suppose $S$ is not usc at $f$. Then for some open sets $V \ni x_{0}, W \ni y_{0}$ there is a sequence of functions $f_{n},\left\|f_{n}-f\right\| \rightarrow 0$, and points $\left(x_{n}, y_{n}\right) \in S\left(f_{n}\right) \backslash(V \times W)$. It is not difficult to see that $\left(x_{n}, y_{n}\right)_{n \geqslant 1}$ is an optimising sequence for $\left(P_{f}\right)$. Well posedness of $\left(P_{f}\right)$ implies that $\left(x_{n}, y_{n}\right)_{n \geqslant 1}$ belongs to $V \times W$ eventually, and this is a contradiction. Let now $S(f)=\left(x_{0}, y_{0}\right)$ and suppose $S$ is usc at $f$. Take some optimising sequence $\left(x_{i}, y_{i}\right)_{i \geqslant 1}$ for $\left(P_{f}\right)$. We shall show that $\left(x_{i}, y_{i}\right)_{i \geqslant 1}$ converges to $\left(x_{0}, y_{0}\right)$. Let $V \ni x_{0}, W \ni y_{0}$ be open sets and $\varepsilon>0$ be such that $S\left(f_{\varepsilon}\right) \subset V \times W$. This is possible because $S$ is usc at $f$. Since $S\left(f_{\varepsilon}\right)$ contains the tail of any optimising sequence we get that $\left(x_{i}, y_{i}\right) \longrightarrow\left(x_{0}, y_{0}\right)$.

Proof of Theorem 4.4: Put $E=C(X \times Y), Z:=G r K$ and $F:=S$. Propositions 4.5, 4.6, 4.7 show that we can apply Theorem 2.7 . The rest of the proof follows from Proposition 4.8.

4.9. Corollary. Let $Y$ be a compact space and let both $X$ and $Y$ contain dense completely metrisable subspaces. Then there exists a dense $G_{\delta}$ subset $G$ of $C(X \times Y)$ such that for every $f \in G$ the problem

$$
\underset{X}{\sup } \inf f(x, y)
$$


is well posed.

Proof: Put $K x=Y$ for every $x \in X$ and apply Theorem 4.4.

Corollary 4.9 generalises the main results from [14] where both $X$ and $Y$ were required to be compact spaces from a proper subclass of the class of spaces containing dense completely metrisable subspaces.

\section{EXAMPLES AND COMMENTS}

A natural question arising in connection with the previous considerations is what happens if $E$ is the space $U(X \times Y)$ of all bounded upper-semicontinuous functions in $X \times Y$. The answer is simple. Regardless of the properties of $X$ and $Y$ the set $E$ is always generically sup-WP.

Proposition 5.1. There exists a dense open subset $U^{*} \subset U(X \times Y)$ such that $\left(P_{f}\right)$ is sup-WP for every $f \in U^{*}$.

Proof: By Proposition 3.3 we know that Dom $M$ is dense in $U(X \times Y)$. Let $x_{0} \in M(f)$, where $f \in U(X \times Y)$. Given $\delta>0$ define

$$
f^{\delta}(x, y)= \begin{cases}f(x, y) & \text { if } x \neq x_{0} \\ f\left(x_{0}, y\right)+\delta & \text { if } x-x_{0}\end{cases}
$$

Clearly, $f^{\delta} \in U(X \times Y)$ and $\left\|f-f^{\delta}\right\| \leqslant \delta$. It is not difficult to see that, for every $h \in U(X \times Y)$ with $\left\|h-f^{\delta}\right\|<\delta / 2, M(h)=\left\{x_{0}\right\}$ and $\left(P_{h}\right)$ is sup-WP.

The conditions (on $X$ and $Y$ ) under which $U(X \times Y)$ is generically well posed are also easy to identify. For simplicity, we shall consider only the case when $K x=Y$ for every $x \in X$. That is, for $f \in E$, we consider the problem

$$
\sup _{X} \inf _{Y} f(x, y)
$$

Theorem 5.2. Let $X$ and $Y$ be topological spaces and $U^{*}=U(X \times Y)$ be the space of all bounded upper semicontinuous functions in $X \times Y$. Then the following statements are equivalent:

(a) $Y$ contains some dense completely metrisable subset;

(b) $U^{*}$ is generically well posed.

Proof: Let $\alpha=\{U\}$ be a maximal disjoint system of open sets $U \subset U^{*}$ such that for every $U \in \alpha$ there is some $x_{u} \in U$ with $\inf _{Y} f\left(x_{u}, y\right)>\sup _{x \neq x_{u}} \inf _{Y} f(x, y)$ whenever $f \in U$. In particular, this means that $M(f)=\left\{x_{u}\right\}$ for every $f \in U$. As above we can prove that $E:=\bigcup\{U: U \in \alpha\}$ is an open and dense subset of $U^{*}$. 
Consider the mapping $F$ which assigns to each $f \in U \in \alpha$ the set

$$
F(f):=\left\{y^{*} \in Y: f\left(x_{u}, y^{*}\right)=\inf _{Y} f\left(x_{u}, y\right)\right\}
$$

The problem of minimising the upper semicontinuous function $f\left(x_{u}, y\right)$ is equivalent to the problem of maximising the lower semicontinuous function $-f\left(x_{u}, y\right)$ and we can prove (as in Section 3) that $F$ is an open and minimal mapping with dense domain on every $U \in \alpha$ and, therefore, on $E$. Hence $F(E)$ is an open subset of $Y$. We shall show now that $F(E)$ is also dense in $Y$. Indeed, take some open $W \neq \emptyset$ in $Y$ and some continuous function $h: Y \rightarrow[0,1]$ such that $h(Y \backslash W)=1$ and $h\left(y_{0}\right)=0$ for some $y_{0} \in W$. Consider the function $f_{0}(x, y):=h(y)$. Evidently, $f_{0} \in U^{*}$. For every $f$ with $\left\|f-f_{0}\right\|<1 / 3$ and every $x_{0} \in X$ we have $\left\{y^{*} \in Y: f\left(x_{0}, y^{*}\right)=\inf _{Y} f\left(x_{0}, y\right)\right\} \subset W$. Since $E$ is dense in $U^{*}$ the set $U^{\prime}:=B\left(f_{0}, 1 / 3\right) \cap E$, where $B\left(f_{0}, 1 / 3\right)$ is the open ball around $f_{0}$, is not empty for some $U \in \alpha$. Clearly $F\left(U^{\prime}\right) \subset W$.

The rest of the proof follows from Theorem 2.7 and Proposition 3.6.

The following two examples exhibit situations when there is no generic well posedness.

EXAmPLE 5.3. Let $X$ be the Cartesian product $\Pi\left\{I_{\alpha}: \alpha \in A\right\}$, where $I_{\alpha}=$ $[0,1]$ for every $\alpha$ from the uncountable set $A$. Then every nonempty $G_{\delta}$ subset of $X$ contains uncountably many points. In particular, every lsc real-valued function $f: X \longrightarrow R$ is not sup-WP.

ProOF: Let $V_{i}, i \geqslant 1$, be open subsets of $X$ and $x_{0}=\left(x_{\alpha}^{0}\right)_{\alpha \in A} \in \bigcap_{i \geqslant 1} V_{i}$. Without loss of generality we can suppose that $V_{i}$ is a basic neighbourhood of the form $V_{i}=$ $\Pi\left\{\left(x_{\alpha_{i}}^{0}-\varepsilon, x_{\alpha_{i}}^{0}+\varepsilon\right): i \in A_{i}\right\} \times \Pi\left\{I_{\alpha}: \alpha \notin A_{i}\right\}$, where $A_{i}$ is a finite subset of $A$. Put $A^{\prime}:=\bigcup A_{i}$. Then the set $\left(x_{\alpha}^{0}\right)_{\alpha \in A^{\prime}} \times \Pi\left\{I_{\alpha}: \alpha \notin A^{\prime}\right\}$ is uncountable and is contained in $\bigcap_{i \geqslant 1} V_{i}$.

EXAMPLE 5.4. Let $N$ be the set of all positive integers with the discrete topology and let $\beta N$ be the Stone - Čech compactification of $N$. Then every nonempty $G_{\delta}$ subset of $X:=\beta N \backslash N$ has uncountably many points.

\section{REFERENCES}

[1] T. Basar and G. Olsder, Dynamic noncooperative game theory (Academic Press, New York, 1982).

[2] G. Beer and R. Lucchetti, 'Convex optimization and the epidistance topology', Trans. Amer. Math. Soc. 327 (1991), 795-814.

[3] G. Beer and R. Lucchetti, 'The epidistance topology: continuity and stability results with applications to convex optimization problems', Math. Oper. Res. 17 (1992), 715-728. 
[4] M.M. Čban and P.S. Kenderov, 'Dense Gàteaux differentiability of the sup norm in C(T) and topological properties of 'T', C.R. Acad. Bulg. Sci. 38 (1985), 1603-1604.

[5] M.M. Čoban and P.S. Kenderov, 'Generic Gâteaux differentiability of convex functionals in $C(T)$ and topological properties of $T$ ', in Mathematics and Education in Mathematics, Proceedings of the XV-th Spring Conference of the Union of Bulgarian Mathematicians, 1986, pp. 141-149.

[6] M.M. Čoban, P.S. Kenderov and J.P. Revalski, 'Generic well-posedness of optimization problems in topological spaces', Mathematika 36 (1989), 301-324.

[7] F. De Blasi and J. Myjak, 'On the minimum distance to a closed convex set in a Banach space', Bull. Acad. Sci. Pol 29 (1981), 373-376.

[8] F. De Blasi and J. Myjak, 'Some generic properties in convex and nonconvex optimization theory', Ann. Soc. Math. Polon. 24(1984), 1-14.

[9] A. Dontchev and T. Zolezzi, Well-posed optimization problems, Lecture Notes in Mathematics 1543 (Springer-Verlag, Berlin, Heidelberg, New York, 1993).

[10] L. Holá, 'Most of the optimization problems have unique solution', C.R. Acad. Bulg. Sci. 42 (1989), 5-8.

[11] J.E. Jane and C.A. Rogers, 'Borel electors for upper semicontinuous set-valued maps', Acta Math. 155 (1985), 41-79.

[12] P.S. Kenderov, 'Most of optimization problems have unique solution', C.R. Acad. Bulg. Sci. 37 (1984), 297-299.

[13] P.S. Kenderov and J.P. Revalski, 'The Banach-Mazur game and generic existence of solution to optimization problems', Proc. Amer. Math. Soc. 118 (1993), 911-917.

[14] P.S. Kenderov and N.K. Ribarska, 'Generic uniqueness of the solution of "min-max" problems', in Lecture Notes in Economics and Mathematical Systems 304 (Springer-Verlag, Berlin, Heidelberg, New York, 1988), pp. 41-48.

[15] M.B. Lignola and J. Morgan, 'Topological existence and stability for minsup problems', J. Math. Anal. Appl. (1990), 165-180.

[16] P. Loridan, 'An application of Ekeland's variational principle to generalized Stackelberg problems', (Written version of a lecture presented to the Workshop on Well-Posedness in Optimization, Santa Margherita, Ligure, 1991).

[17] P. Loridan and J. Morgan, ' $\varepsilon$-regularized two-level optimization problems; approximation and existence results', in Lecture Notes in Mathematics 1405 (Springer-Verlag, Berlin, Heidelberg, New York, 1989), pp. 99-113.

[18] R. Lucchetti and F. Patrone, 'Sulla densitàe genericità di alcuni problemi di minimimo ben posti', Boll. Un. Mat. Ital. B 15 (1978), 225-240.

[19] J. Morgan, 'Constrained well-posed two-level optimization problems', in Nonsmooth optimization and related topics, (F. H. Clarke, V. F. Dem'yanov and F. Giannessi, Editors) (Plenum Press, New York and London, 1989).

[20] I. Namioka, 'Radon-Nikodým compact spaces and fragmentability', Mathematika 34 (1987), 258-281.

[21] F. Patrone, 'Most convex functions are nice', Numer. Funct. Anal. Optimi. 9 (1987), 359-369. 
[22] J.P. Revalski, 'Generic well-posedness in some classes of optimization problems', Acta Univ. Carolin. Math. Phys. 28 (1987), 117-125.

[23] N.K. Ribarska, 'Interval characterization of fragmentable spaces', Mathematika 34 (1987), 243-257.

[24] H. Von Stackelberg, The theory of market economy (Oxford University Press, Oxford, 1952).

[25] A. Tyhonov, 'On the stability of the functional optimization problem', USSR Comput. Math. and Math. Phys. 6 (1966), 28-33.

[26] T. Zolezzi, Well posed optimal control problems: A perturbation approach, IMA Proceedings (Springer Verlag, Berlin, Heidelberg, New York, to appear).

[27] T. Zolezzi, 'Well posedness of optimal control problems', (Preprint No 236, Dipartimento di Matematica Universita di Genova, 1993).

Institute of Mathematics

Bulgarian Academy of Sciences

Acad. G. Bonchev Street

1113 Sofia

Bulgaria

\author{
Department of Mathematics \\ University of Milano \\ via Saldini 50 \\ 20133 Milano \\ Italy
}

